\title{
Entanglement-enhanced two-photon delocalization in a coupled-cavity array
}

\author{
Shi-Qing Tang, Ji-Bing Yuan, Xin-Wen Wang, and Le-Man Kuang ${ }^{* \dagger 1}$ \\ ${ }^{1}$ Key Laboratory of Low-Dimensional Quantum Structures and Quantum Control of Ministry of Education, \\ and Department of Physics, Hunan Normal University, Changsha 410081, China
}

\begin{abstract}
We study transport properties of two entangled photons which are initially injected into two nearest-neighbor coupling cavities in a one-dimensional coupled-cavity array (CCA). It is found that photonic transport dynamics in the two-photon CCA exhibits entanglement-enhanced two-photon delocalization (TPD) phenomenon. It is shown that the CCA can realize the localization-to-delocalization transition for two entangled photons.
\end{abstract}

PACS numbers: 03.67.-a, 42.50.Pq, 37.30.+i

\section{INTRODUCTION}

In recent years, much attention has been paid to coupledcavity arrays (CCAs) [1-10] due to their extremely rich physics as well as their wide potential applications to work as an effective platform to realize quantum information processing and to carry out photonics tasks. For instance, CCAs stand out as an attractive controllable test bed for strongly correlated many-body models and quantum simulators of many-body physics [1-5, 11, 12]. Transport properties of photons and various excitations in CCAs embedded with natural or artificial atoms are also receiving considerable interest [13-24]. In particular, it has been demonstrated that CCAs with a cyclic three-level system or a single atom open the possibility to quantum routing of single photons [25, 26].

CCAs typically consist of an arrangement of low-loss cavities with nearest-neighbor coupling allowing photon hopping between neighboring cavities. An appealing feature of CCAs is the available high control of each single cavity in terms of performable measurements, quantum-state engineering, and dynamical parameter tuning. This feature can be used to realize local addressing in quantum information processing. Progress in the fabrication techniques make CCAs experimentally accessible [27-29]. Although quantum entanglement is an essential resource in quantum information processing, propagation of spatially entangled photons in CCAs without natural or artificial atoms has not been studied extensively heretofore. A natural question is how quantum entanglement of initial input photons affects photonic transport properties in CCAs.

In this paper, we study transport properties of two initially entangled photons which are injected into two nearestneighbor coupling cavities in a one-dimensional CCA. We show that photonic transport dynamics in the two-photon CCA exhibits the entanglement-enhanced two-photon delocalization (TPD) phenomenon. We investigate TPD dynamics by introducing the concept of the TPD degree when two nearest-neighbor coupling cavities are initially in twophoton entangled state $|\Psi\rangle=\sin \theta|2,0\rangle+\cos \theta|0,2\rangle$. We demonstrate that the CCA can realize the localization-todelocalization transition for two entangled photons.

The rest of this paper is organized as follows. In Sec II, we present our physical model and its solution. In Sec III, we analyze photonic transport properties and TPD in the two-photon CCA, and show the entanglement-enhanced

* Author to whom any correspondence should be addressed.

†Email: lmkuang@hunnu.edu.cn
TPD and the localization-to-delocalization transition for two entangled photons. Finally, we shall conclude our paper with discussions and remarks in the last section.

\section{PHYSICAL MODEL AND SOLUTION}

Let us consider a finite-length CCA in which nearestneighbor cavities are coupled together so as to allow for photon hopping. We assume that all the cavities are identical. Each cavity sustains a single field mode of frequency $\omega$. The Hamiltonian of the full system (here and throughout we take $\hbar=1)$ is written as

$$
\hat{H}=\omega \sum_{j=1}^{N} a_{j}^{\dagger} a_{j}+J \sum_{j=1}^{N-1}\left(a_{j}^{\dagger} a_{j+1}+H . c .\right),
$$

where $\hat{a}^{\dagger}(\hat{a})$ is a photonic creation (annihilation) operator at $i$-th cavity. The second term in above equation represents the hopping interaction with the intercavity coupling strength $J$.

The Hamiltonian of the CCA given by Eq. (1) can be diagonalized in terms of the normal modes defined by

$$
\hat{c}_{k}(t)=\sum_{j=1}^{N} \hat{a}_{j}(t) S(j, k)
$$

with the following inverse transformation

$$
\hat{a}_{j}(t)=\sum_{k=1}^{N} \hat{c}_{k}(t) S(j, k),
$$

where the transformation matrix is given by

$$
S(j, k)=\sqrt{\frac{2}{N+1}} \sin \left(\frac{j \pi k}{N+1}\right) .
$$

Making use of Eqs. (2)-(4), one can obtain the diagonalized form of Hamiltonian (1) where the transformation matrix is given by

$$
\hat{H}=\sum_{k=1}^{N} \Omega_{k} c_{k}^{\dagger} c_{k}, \quad \Omega_{k}=\omega+2 J \cos \left(\frac{\pi k}{N+1}\right) .
$$

From Heisenberg equations on Eq. (5) and making use of Eq. (3), one can obtain exact solution of the CCA in the heisenberg representation with the following form

$$
a_{j}(t)=\sum_{l} G_{j l}(t) a_{l}(0)
$$


where the explicit form of the Green's function is given by

$$
G_{j, l}(t)=\sum_{k=1}^{N} \exp \left\{-i \Omega_{k} t\right\} S(j, k) S(l, k) .
$$

In the following section, we shall use above exact solution to investigate quantum dynamics of photonic transport and TPD in the two-photon CCA.

\section{ENTANGLEMENT-ENHANCED TWO-PHOTON DELOCALIZATION}

The quantum mechanical properties of light are observed when correlations between the propagating photons are considered. In this section, we study photonic transport properties and quantum dynamics of two-photon delocalization in the two-photon CCA when two entangled photons are injected into the CCA by investigating the evolution of the photon-number correlation function

$$
P_{m, n}(t)=\left\langle a_{n}^{\dagger}(t) a_{m}^{\dagger}(t) a_{m}(t) a_{n}(t)\right\rangle,
$$

which represents the probability of detecting a two-photon coincidence across cavity $m$ and cavity $n$. The probability to detect both photons at the same cavity $n$ is given by $P_{n, n}(t) / 2$.

We consider such a situation in which the two photons are initially in $r$-th cavity and $s$-th cavity with the following twophoton NOON-type states

$$
|\psi\rangle=\sin \theta|2\rangle_{r}|0\rangle_{s}+\cos \theta|0\rangle_{r}|2\rangle_{s},
$$

where for the simplicity, we take $0 \leqslant \theta \leqslant \pi / 2$ throughout the paper. It is interesting to note that the two photons in the two-photon entangled states (9) are in the same cavity, i.e., cavity $r$ or cavity $s$. The other cavities in the CCA are in vacuum state. Hence, the two-photon entangled states given by Eq. (9) are two-photon localization states. The amount of entanglement of the two-photon states in Eq.(9) can be measured by concurrence [30] with the following expression

$$
\mathcal{C}(\theta)=|\sin 2 \theta| \text {. }
$$

Obviously, the concurrence vanishes when $\theta=0$, and $\pi / 2$ while the concurrence reaches the maximum value 1 when $\theta=\pi / 4$.

Substituting Eqs. (6) and (9) into Eq. (8), we can obtain the probability of detecting a two-photon coincidence across cavity $m$ and cavity $n$ with the following expression

$$
P_{m n}=2\left|\cos \theta G_{m r}(t) G_{n r}(t)+\sin \theta G_{m s}(t) G_{n s}(t)\right|^{2} .
$$

As an example, we consider the photonic propagation in the CCA for the two-photon entangled state given by Eq. (9) which is injected initially into two neighboring cavities, say $r=15$ and $s=16$. Assume that the CCA under our consideration consists of 29 cavities $(N=29)$. Quantum evolution of the calculated correlation function for this case is presented in Fig. 1 for different amount of initial entanglement. Fig.1(a), Fig.1(c), and Fig.1(e) are the initialstate correlation function when the amount of initial entanglement $\mathcal{C}=0,0.5$, and 1, respectively. Fig.1(b), Fig.1(d), and Fig.1(f) are the correlation function at time $\omega t=83.57$
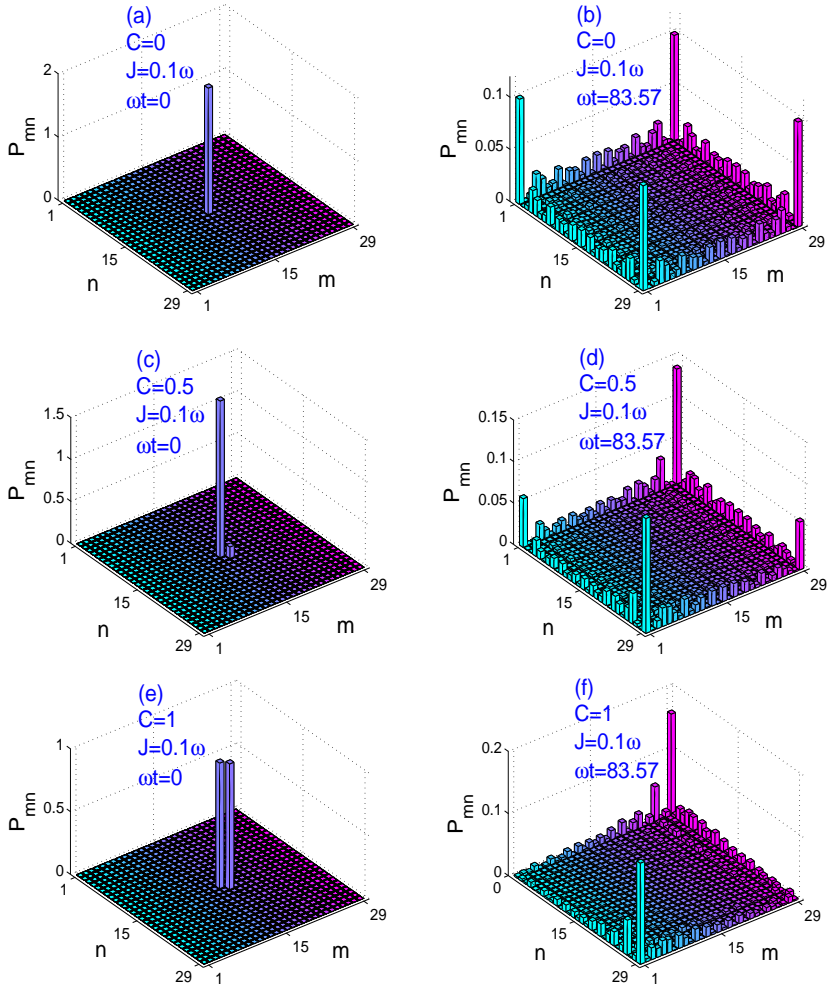

FIG. 1: (Color online) The joint probability $P_{m n}$ of simultaneously detecting photons in cavity $m$ and $n$ after a temporal evolution $\omega t=83.57$. The evolution time is scaled by $1 / J$, and other parameters are $\omega=J, N=29$.

when the amount of initial entanglement $\mathcal{C}=0,0.5$, and 1 , respectively. From Fig. 1 we can see that the correlation represented by the diagonal elements of the two-photon correlation function $P_{m n}$ completely vanishes while the correlation represented by the off-diagonal elements of the two-photon correlation function $P_{m n}$ is enhanced with the increase of the initial amount of entanglement from vanishing entanglement $(\mathcal{C}=0)$ to the maximal entanglement $(\mathcal{C}=1)$. Accordingly, the two photons will always tend to separate and to emerge in different cavities.

In order to well understand transport dynamics of the two photons in the CCA, we introduce the concept of the twophoton delocalization (TPD). When the two photons are in two different cavities in the two-photon CCA we call it as the TPD while when the two photons are in the same cavity we call it as the two-photon localization (TPL). The degree of the TPD can be defined in terms of the sum of probability $P_{n n} / 2$ of detecting photons in every cavity

$$
\eta=1-\frac{1}{2} \sum_{n=1}^{N} P_{n, n}(t)
$$

which represents the probability of the two photons being not at the same cavity in the CCA simultaneously. Especially, when $\eta=1$, the two photons are in the complete TPD state in which the two photons are completely in different cavities. In this case, it is impossible that the two photons are in the same cavity. Hence, the two photons in the TPD state tend to stay at different cavities. In this sense, the TPD degree can reflect repulsive-statistics or anti-bunching property of two photons in the CCA. Obviously, the TPD degree of the two-photon NOON-type state defined by Eq. (9) is 


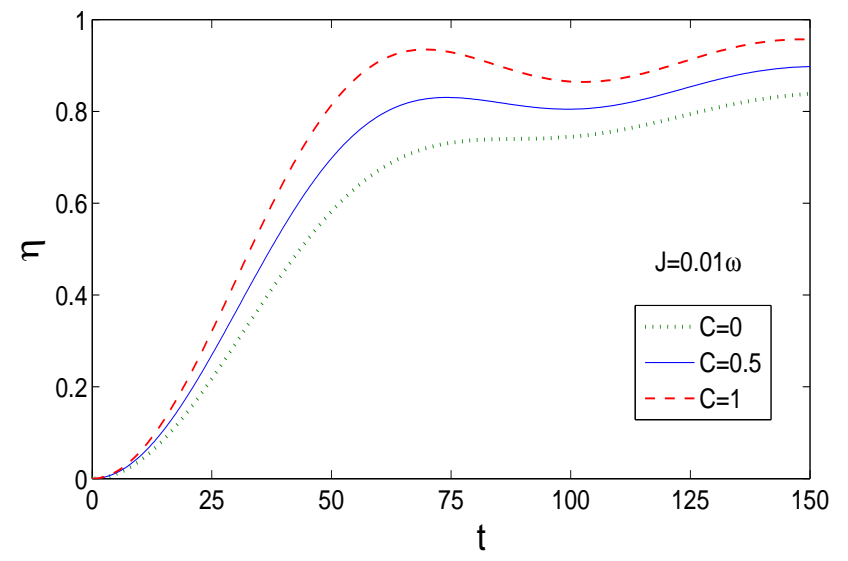

FIG. 2: (Color online) Time evolution of the two-photon delocalization degree $\eta$ in the weak hopping regime for three different values of quantum entanglement of the two-photon state given by Eq. (9). The evolution time is scaled by $1 / J$. The photon hopping parameter takes $J=0.01 \omega$. The number of the cavities in the CCA is $N=29$. The dashed curve represents the result of the maximally entangled state with $\mathcal{C}=1$, the solid line corresponds to the initial input state with the concurrence $\mathcal{C}=1$, and the dot line shows the result of the initial unentangled state with $\mathcal{C}=0$.

zero. Hence, the state given by Eq. (9) is not a TPD state, it is a TPL state. The larger the value of the parameter $\eta$ is, the stronger the TPD phenomenon becomes. We consider the two-photon CCA formed by 29 cavities. When the two photons are injected into 15-th and 16-th cavities with the input state (9), the photon-number second-order correlation function changes considerably in the time evolution as shown in Fig. 1. The most obvious feature is the appearance of the TPD in which the diagonal elements of the two-photon probability, i.e., $P_{n n} / 2$, are suppressed considerably in the time evolution. In particular, for the case of the maximally entangled state with the concurrence $\mathcal{C}=1$ we can observe almost completeTPD with $P_{n n} \approx 0$ in the process of the time evolution as shown in Fig. 1(e) and Fig. 1(f).

We now investigate quantum dynamics of the TPD and the influence of quantum entanglement of the initial state in the weak hopping regime of $J \ll \omega$. In Fig. 2, we have plotted the time evolution of the TPD degree for three different values of quantum entanglement of the initial state (9), $\mathcal{C}=, 0,0.5$, and 1 , respectively. We here take the photon hopping strength $J=0.01 \omega$ and the number of the cavities in the CCA $N=29$. The dashed curve represents the case of the maximally entangled state with $\mathcal{C}=1$, the solid line corresponds to the initial input state with the concurrence $\mathcal{C}=0.5$, and the dot line represents the case of the initial unentangled state with $\mathcal{C}=0$. From Fig. 2 we can see that the two photons in the CCA rapidly tend to the TPD with the time evolution in a short-time regime. This clearly indicates the transition from the TPL state to the TPD state in the two-photon CCA. The TPD degree can be preserved in the process of the long time evolution afterwards except some small oscillations. On the other hand, Fig. 2 indicates that the TPD is enhanced with the increase of quantum entanglement of the initial state (9). Hence, we can conclude that quantum entanglement can essentially affect the TPD.

We then turn to quantum dynamics of the TPD in the strong hopping regime of $J \approx \omega$. In. Fig. 2, we have plot-

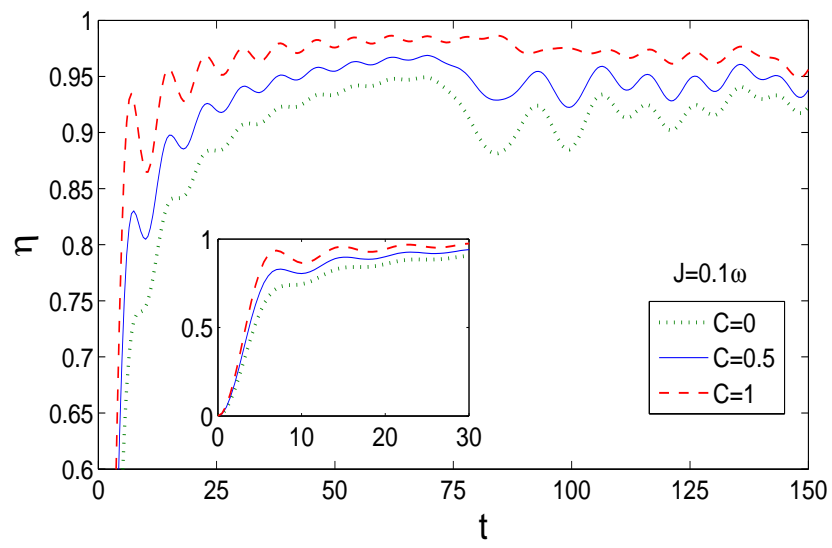

FIG. 3: (Color online) Time evolution of the two-photon delocalization degree $\eta$ in the strong hopping regime for three different values of quantum entanglement of the two-photon state given by Eq. (9). The evolution time is scaled by $1 / J$. The photon hopping parameter takes $J=0.1 \omega$. The number of the cavities in the CCA is $N=29$. The dashed curve represents the result of the maximally entangled state with $\mathcal{C}=1$, the solid line corresponds to the initial input state with the concurrence $\mathcal{C}=1$, and the dot line shows the result of the initial unentangled state with $\mathcal{C}=0$.

ted the time evolution of the TPD degree $\eta$ for three different values of quantum entanglement of the initial state $|\psi\rangle$. The photon hopping parameter takes $J=0.1 \omega$. The number of the cavities in the CCA is $N=29$. The dashed curve represents the case of the maximally entangled state with $\mathcal{C}=1$, the solid line corresponds to the initial input state with the concurrence $\mathcal{C}=1$, and the dot line represents the case of the initial unentangled state with $\mathcal{C}=0$. From Fig. 3 we can clearly observe the entanglement-enhanced TPD phenomenon. In comparison with the quantum dynamics of the TPD in the weak hopping regime of $J \ll \omega$, from Fig.2 and Fig. 3 we can see that the TPD in the strong hopping regime becomes faster than that in the weak hopping regime in the short time regime. The oscillations of the TPD degree in the process of the time evolution become stronger with the increasing the photon hopping strength. However, the TPD degree is over 90 percent in the most time. The short time behavior of the TPD degree is indicated in the inset in Fig. 3 , from which we can see that the transition from the TPL to the TPD happens in shorter time in the strong hopping regime than the weak hopping regime.

\section{CONCLUDING REMARKS}

In conclusion, we have studied photonic transport properties and TPD dynamics in the two-photon CCA when two entangled photons are initially injected into two nearestneighbor coupling cavities in the form of two-photon NOON-type states which are superposition states of twophoton states $|2,0\rangle$ and $|0,2\rangle$. We have investigated the TPD dynamics by introducing the concept of the TPD degree for the initial two-photon entangled states $|\psi\rangle=\sin \theta|2,0\rangle+$ $\cos \theta|0,2\rangle$. It has been found that photonic transport dynamics in the two-photon CCA exhibits the entanglementenhanced TPD phenomenon. It has demonstrated that the CCA can realize the localization-to-delocalization transition 
for two entangled photons. It is worthwhile to point out that the entanglement-enhanced TPD phenomenon is a twophoton cooperative effect induced by quantum interference of two entangled photons due to the initial quantum entanglement. The entanglement-enhanced TPD vanishes in the absence of entanglement between two photons.

\section{Acknowledgments}

This work was supported by the National 973 Program under Grant No. 2013CB921804 and the NSF under Grant No. 11375060 and Grant No. 11434011.
[1] Illuminati F 2006 Nat. Phys. 2803

[2] Greentree A D, Tahan C, Cole J H and Hollenberg L C L 2006 Nat. Phys. 2856

[3] Hartmann M J, Brandão F G S L and Plenio M 2006 Nat. Phys. 2849

[4] Hartmann M J, Brandão F G S L and Plenio M 2007 Phys. Rev. Lett. 99160501

[5] Hartmann M J, Brandão F G S L and Plenio M 2008 Laser Photon. Rev. 2527

[6] Tomadin A and Fazio R 2010 J. Opt. Soc. Am. B 27130

[7] Houck A, Tureci H and Koch J 2012 Nat. Phys. 8292

[8] Schmidt S and Koch J 2013 Ann. Phys. 525395

[9] Mlmeida G M A and Souza A M C 2013 Phys. Rev. A 87 033804

[10] Lombardo F, Ciccarello F, and Palma G M 2014 Phys. Rev. A 89053826

[11] Greentree A D, Tahan C, Cole J H and Hollenberg L C L 2006 Nat. Phys. 2856

[12] Angelakis D G, Santos M F and Bose S 2007 Phys. Rev. A 76 031805(R)

[13] Zhou L, Lu J and Sun C P 2007 Phys. Rev. A 76012313

[14] Hu F M, Zhou L, Shi T and Sun C P 2007 Phys. Rev. A 76 013819

[15] Zhou L, Gao Y B, Song Z and Sun C P 2008 Phys. Rev. A 77 013831

[16] Ogden C D, Irish E K and Kim M S 2008 Phys. Rev. A 78 063805

[17] Makin M I, Cole J H, Hill C D, Greentree A D and Hollenberg

\section{C L 2009 Phys. Rev. A 80043842}

[18] Bose S, Angelakis D G and Burgarth D 2007 J. Mod. Opt. 54 2307

[19] Quach J, Makin M I, Su C H, Greentree A D and Hollenberg L C L 2009 Phys. Rev. A 80063838

[20] Paternostro M, Agarwal G S and Kim M S 2009 New J. Phys. 11013059

[21] Longo P, Schmitteckert P and Busch K 2010 Phys. Rev. Lett. 104023602

[22] Gong Z R, Ian H, Zhou L and Sun C P 2008 Phys. Rev. A 78 053806

[23] T. Shi and Sun C P 2009 Phys. Rev. B 79205111

[24] Liao J Q, Gong Z R, Zhou L, Liu Y X, Sun C P and Nori F 2010 Phys. Rev. A 81042304

[25] Zhou L, Yang L P, Li Y and Sun C P 2013 Phys. Rev. Lett. 111 103604

[26] Lu J, Zhou L, Kuang L M and Nori F 2014 Phys. Rev. A 89 013805

[27] Wallraff A, Schuster D, Blais A, Frunzio L, Huang R, Majer J, Kumar S, Girvin S and Schoelkopf R 2004 Nature 431162

[28] Hennessy K, Badolato A, Winger M, Gerace D, Atatüre M, Gulde S, Fält S, Hu E L and Imamoğlu A 2007 Nature 445 896

[29] Faraon A, Fushman I, Englund D, Stoltz N, Petroff P, and Vuckovic J 2008 Nat. Phys. 4859

[30] Wootters W K 1998 Phys. Rev. Lett. 802245 